\title{
Article
}

\section{Developing team decision-making: A holistic framework integrating both on- field and off-field pedagogical coaching processes}

Richards, Pamela, Collins, David John and Mascarenhas, D.R.D.

Available at http://clok.uclan.ac.uk/15895/

Richards, Pamela ORCID: 0000-0003-4242-981X, Collins, David John ORCID: 0000-0002-7601-0454 and Mascarenhas, D.R.D. (2017) Developing team decision-making: A holistic framework integrating both on-field and off-field pedagogical coaching processes. Sport Coaching Review, 6 (1). pp. 57-75. ISSN 2164-0629

It is advisable to refer to the publisher's version if you intend to cite from the work. http://dx.doi.org/10.1080/21640629.2016.1200819

For more information about UCLan's research in this area go to http://www.uclan.ac.uk/researchgroups/ and search for <name of research Group>.

For information about Research generally at UCLan please go to http://www.uclan.ac.uk/research/

All outputs in CLoK are protected by Intellectual Property Rights law, including Copyright law. Copyright, IPR and Moral Rights for the works on this site are retained by the individual authors and/or other copyright owners. Terms and conditions for use of this material are defined in the policies page. 
7

8

9

10

Developing team decision making: A holistic framework integrating both on-field and off-field pedagogical coaching processes.

Pam Richards ${ }^{1}$, Dave Collins ${ }^{2}$ and Duncan R. D. Mascarenhas ${ }^{1}$

1. Department of Sport and Exercise Science Wrexham Glynd ŵr University, UK

2. Institute of Coaching and Performance, University of Central Lancashire

Second Resubmission $5^{\text {th }}$ June May 2016

Correspondence concerning this article should be addressed to:

Pam Richards

Wrexham Glyndwr University

Department of Sport and Exercise Science

Plas Coch Campus

Mold Road

Wrexham, LL12 2AW

Phone: +44 (0) 1978365367

Mobile:07950909229

Email: pam.richards@me.com 


\section{Abstract}

This paper explores the complexity of developing decision-making skills in team

sports. Research from the domains of motor control, perception and pedagogy (Teaching Games for Understanding and Game Sense) has significantly enhanced our knowledge of decision-making in sport. However, such studies although contributing knowledge, have explored elements of decision-making in isolation and many have failed to consider the naturalistic context in which decisions are 


\section{Introduction}

It has been well documented that perception is vital to the development of decision making (hereafter DM) in team sports (Williams, 2009). Unfortunately, limited consideration has been directed towards exploring the influence of the playing context in which such decisions are made (cf. Bar-Eli, Plessner, \& Raab, 2011) and the significant role of the player's own subjective, cognitively-based perception relating to these situations. Consequently, coaches need to appreciate how the playing context influences what information players perceive, attend to and prioritise whilst playing.

Our point here is that 'mainstream' sport science research seems to have addressed too narrow a band of the factors which, through complex interplay, enable the DM process to take place in high pressure, time limited settings. Moreover this is often performed with initially disparate groups of players in ways that seek to gain their commitment, buy-in and, eventually cognitive investment to build a shared understanding. For example, perceptual elements are clearly crucial, and a substantial literature has addressed this in sports settings. There is a comparative dearth of research however, which shows how individual perceptions can be made more similar and, even more crucially, how these more common perceptions can be used to generate common but effective DM strategies and outcomes. Simply put, coaches seem to be waiting for science to address what is a central but neglected concern.

Of course, DM in team sports is much more than a collection of separate activities (Williams, 2009). Rather, it requires a complex and dynamic integration of several elements and processes, which simultaneously and dynamically interact. These processes include the interaction between situational/tactical aspects (e.g., teammates, opposition, area of the pitch) and strategic factors (team philosophy and match objectives), both of which must be similarly applied by all team members, often without any overt in-game communication, through the 
development and application of a Shared Mental Model or SMM (Richards, Collins, \& Mascarenhas, 2012).

\section{Developing team DM: What do we know and what do we need?}

Confusion still exists amongst practitioners and researchers with regard to the precise mechanisms which should be used to develop expert decision makers within applied sporting contexts. Williams (2009) recognises the valuable contribution of existing research in the area of DM, but suggests that investigating perceptual skills in isolation of the performance setting may have inflated the contribution these make in competition.

Of course, we acknowledge that the motoric and perceptual elements of technique are fundamental to the development of DM skills in team sports. A significant amount of research has identified the perceptual cognitive skills which are essential to the DM process (Starkes \& Ericson, 2003; Williams, 2009). Such research informs us about a wide range of factors, including what cues skilled performers use to assess the opposition's movements (Williams, 2009), what stimuli are identified in recognising play patterns (Klein, 1993; Williams 2009) and at what stage this information is attended to in dynamic play situations (North \& Williams, 2008). In addition, research has highlighted the importance of visual search behaviours in skilled performers (Williams, 2009) and the need to use cue identification and anticipation to inform DM (Caserta, Young, \& Janelle, 2005; Williams, 2009). Other crucial elements have been comparatively neglected, such as how commonality of perception is encouraged and how this information, once established, can best be used to generate optimum styles of DM through essential cognitive processes, both within individuals and across teams. As a vital next step therefore, practitioners need to take responsibility for understanding and designing training environments that integrate the player's subjective cognitive perceptions of the situations within the context of the performance setting. 
The development of high quality team decision makers therefore appears to be more

102

103

104

105

106

107

108

109

110

111

112

113

114

115

complex than just facilitating a perceptual recognition or problem solving approach in training environments (although both of these significantly enhance our understanding of the process). Numerous theories relating to DM exist (see Bar-Eli et al., 2011) but only a limited number explore the process of DM in the sporting context. Each of these theories provides a valuable contribution to our understanding of the DM process in sport. However, most explore an aspect of DM in isolation and ignore the complexity of the dynamic competitive situations in which several components interact.

We propose that the nature of DM in invasion sports lends itself to the Naturalistic Decision Making (NDM) paradigm, where decisions in sport are performed in complex and often unpredictable conditions, in pressurised situations and with extreme time constraints (Klein, 2008). NDM researchers seek to investigate how experts perform tasks in dynamic environments which have ill-structured problems, shifting and changing objectives, time constraints, include multiple players and are influenced by organisational goals. All these characteristics are representative of high performance team sport environments. Unfortunately, such a real world approach, heavily laden with contextual information, has often been neglected in perception-action research where such information has a bidirectional link between perceptions and actions (Beilock, 2009). Accordingly, this paper will explore the specific NDM theories of Recognition Primed Decision making (RPD), Situational Awareness (SA) and sensemaking (Klein, Phillips, Rall \& Peluso, 2007) in context of the proposed framework (see Figure 1 and 2). Each approach makes a valuable and distinctive contribution individually but when collectively integrated, they provide a comprehensive justification of the possible mechanisms through which DM might be developed in sport. Supporting our integration of these constructs within a single framework, Bar-EIi et al. (2011) proposed that our understanding of the DM process will only be improved by applying theories directly into the sporting environment. The authors suggest that specific situations 
may require the development of specialised models that can be applied to the dynamic context of the sporting setting (Richards et al, 2012).

As applied practitioners working with elite sports teams developing DM skills, we propose that there are three factors that are worthy of consideration. Firstly, in examining the context of the situation, we need to be cognisant of the unique characteristics of the team, such as tactical plans and the team's philosophy; such aspects guide the application of the team developing a SMM (Richards et al., 2009, 2012). Secondly, presuming that team players are attending to, perceiving and valuing elements of the performance display in a similar fashion, then subsequently such team philosophy and game plans will shape players' actions thus directing what information players then attend to. Thirdly the literature needs to present, test and refine DM models and explore how DM characteristics can best be developed in team players. This paper proposes that team DM is developed by layering information (Figure 1) using two dual processes (Figure 2) which illustrates the interaction between an off-field reflective environment and the on-field in action training and competitive environments.

The framework highlights how individual cognitive thought processes can be collectively developed in a progressive manner to establish a collective mind set (Weick \& Roberts, 1993) and develop shared mental models (Oranasanu \& Salas, 1993) of performance which can then be effectively communicated on the field of play. The complexity of developing team decision making is too dynamic and multifactorial to be illustrated singularly in one simple diagram. The framework proposed in this paper consists of two interconnected models. We believe it is only when elements relating to DM are explored holistically in a connected manner can we truly understand the process of developing team DM. The holistic elements manifest themselves in several ways. For the moment, consider the importance of combining perceptual and decision making elements of motor control, the social support generated within a team by self-constructed elements such as key terms and the integration of these within a practical and effective model. All these elements are combined in the approach we 
describe, leading to the use of the term holistic as a hopefully justifiable description. The reader is referred to Richards et al (2012) for an illustrated example of the framework being applied to an international netball team.

\section{The two model process - How each contributes}

Model 1 illustrates the psychomotor processes which are required to develop a shared team cognitive thought process, which itself is positioned within the context of the team's playing philosophy. We advocate that developing team DM skills requires pedagogical process to extend beyond enhancing individual cognitions to integrate the development of shared team cognitions (Oranasanu \& Salas, 1993) and the development of a connected thought process (Weick \& Roberts, 1993). Such processes require information to be seen and valued in the same way by all team members, resulting in the construction of SMMs of performance. Player's individual cognitive thought process are collectively co-ordinated through the pedagogical process of layering information outlined in Model 1, which facilitates members of the team valuing, perceiving and interpreting information in the same connected manner. The ability of all team members to perceive and value dynamic playing situations in the same way, facilitates key information being more effectively attended to and communicated. Model 1 therefore outlines the concepts which necessitate the development of five layers of information which are required to develop team DM (see Figure 1 - what to coach). Each of the layers involves feedback and feed-forward mechanisms, facilitating a cyclical process for continual learning and development of playing constructs. Although illustrated separately to provide clarification for the reader, the five phases continually interact and define each other. Through the process of player empowerment, each of the layers addresses the development of cognitive structures (RPD), mental models (MM) and shared mental models (SMM) and the contextualisation of these structures in the specific environmental situations (Situational Awareness). 
Model 2 in the second section of the paper illustrates the pedagogical process involved in

co-ordinating individual perceptual representations of playing situations, so a collective team cognitive thought process can be obtained. The second model (Figure 2 - how to coach) demonstrates an empirically tested framework that illustrates the interaction between the slow deliberate reflective off-field environment and the rapid on-field competitive environment with the focus of developing team DM. We argue that team DM is developed through a combination of two very different DM paradigms. A slow deliberate reflective off-field training context (more akin to Classical Decision Making; CDM) where mental models are constructed and which simultaneously connects to the in-action high pressurised on-field environment (aligned more with the NDM theoretical approach; see Klein et al, 2007); where mental models are applied and used to inform in action decisions collectively as a team (Merola \& Richards, 2010; Richards et al., 2012). It is proposed that team decision making requires the complex interaction of psychomotor (e.g. technical execution, cue identification, interpretation of situational information and physical movement etc.) and psychosocial processes (e.g. creating of a shared vision and common language amongst coaches and players within the context of shared team philosophy). The creation of pedagogical processes which address psychomotor and psychosocial mechanisms are outlined in Model 1 and 2 and results in the effective identification, interpretation and communication of key information in competitive situations, eventuating in successful team play.

\section{Model 1- Developing decision-making in team sports: Cognitive layering and} contextualisation

Model 1 proposes that the complexity of improving and developing DM skills in team sports demands that we consider the integration of three components; the technical skills required to be executed, the tactical understanding of the play when performed in real-time; and the coordination of all of these aspects by players collectively as a team; all within the particular context of the situation in which the decision is framed (Richards et al., 2012). 
205

206

207

208

209

210

211

212

213

214

215

216

217

218

219

220

221

222

223

\section{Developing a performance vision: Layer 1.}

The initial phase of the model (Figure 1) involves the establishment of the performance vision (which includes team trademarks and generation of concepts) which contribute to the team's playing philosophy. The establishment of the team's concepts is crucial as these provide the framework that will direct attentional focus and determine how knowledge is clustered (Merola \& Richards, 2010). Constructing these concepts in this early phase is initially shaped by the coach's vision of what the 'ideal' performance will ultimately look like for this particular set of players (an alpha version of the performance vision, see Richards et al., 2012). Only when this alpha version of performance is established can it be divided into small aspects of play: for example, an attacking centre pass in netball or an attacking pattern of play in hockey.

During the sporting programme, the coach's vision of performance is reshaped as players are actively encouraged and empowered to contribute to the performance vision (Bate \& Richards, 2011; Merola \& Richards, 2010). The incorporation of the player's perspective (bottom-up approach; Richards et al., 2009; 2012) reshapes the coach's initial vision (alpha version) and results in the construction of the new final beta version. This refinement process is crucial in gaining buy-in from players and will facilitate a deeper and more meaningful engagement with the DM process. The outcome of which is the establishment of a shared team perception, shared language and collective thinking; players are genuinely empowered as they are working within a structure which they helped to create.

This collective performance vision (beta version) results in the construction of MMs that represent key aspects of performance. Mental models have been simply defined as '... internal representations of the external world... [which] represent the experts understanding of the situation' (Serfaty, MacMillan, Entin, \& Entin, 1997, p.235). They provide information about a situation to initially direct attention, then rapidly classify the information and interpret meaning 
from it (Rouse et al., 1992). MMs are only valuable to the individuals who construct them (Westbrook, 2006) so it is important that coaches facilitate opportunities for players (both individually and collectively) to contribute to the development of MMs and subsequently SMMs (Eccles \& Tenenbaum, 2004). The development of a team specific, SMM would demonstrate a collective understanding of a situation in which players are able to execute the specific roles in a coordinated manner to achieve the same perceived outcome (Richards et al., 2009).

Serfaty et al. (1997) elaborate on the concepts of MMs stating that '...expert memory consists of an array of patterns with information items grouped and indexed by their relevance for problem solving in the domain of expertise' (p. 235). This perhaps accounts for the difference between the tactical MMs of an advanced sport performer and those of a novice. The creation of such cognitive internalised structures by players (Richards et al., 2009) provides a framework to structure, order and prioritise relevant information, thus facilitating correct decisions. The formulation of these MMs also enables individuals to learn and comprehend the nature of the situation more quickly (Ross, Battaglia, Phillips, Domeshek, \& Lussier, 2003).

The establishment of the shared performance vision enables information to be transferred and integrated from a top-down knowledge process (alpha performance vision) and a bottom-up knowledge process (MMs and SMMs - developed by players and coaches) simultaneously (Richards et al., 2009). It is these two interactive processes, which are instrumental in shaping the development of DM.

The top-down knowledge process provides the framework in which the beta version of performance can be subdivided into relevant playing aspects. This division enables aspects of performance to be identified and split into more manageable chunks and priorities (e.g., attacking play patterns might be a priority). These key aspects of performance (each one relating to the performance vision) are then rebuilt through the bottom-up knowledge process. Such MMs 
incorporate players' skill sets, individual roles, team principles of play and recognition of situational factors. This process enables players to cluster information and construct internalised plans (Bates \& Richards, 2011; Richards et al., 2012), which facilitate improved DM at an individual, unit and team level.

\section{Perceptual drive and technical execution: Layer 2.}

Technical executions have a direct link to both tactical and strategic layers of the DM process. There is a complex exchange of knowledge and information across the respective knowledge layers, which dictate that several cognitive processes are occurring simultaneously. Shared MMs are driving a top-down approach, influencing cue identification. In this process, players' commonly agree a weighting scale for which factors in any display are the most pertinent. Simultaneously, perceptions of environmental cues (bottom-up) are governing the recognition of tactical patterns (utilising experience), which, in turn are determining what technique will be executed by the player. This process is complex but of relevance here, as the process by which cues are recognised and interpreted determines the action (preparatory movement) taken by individual players prior to the execution of technical skills, therefore resulting in superior skill execution. Notably, Starkes, and Ericsson (2003) confirmed that the way in which technical and tactical skills interact is complex and not well understood.

Importantly, the development of sport skills requires two-stages (Dunn, 2006). This includes the player acquiring a range of technical skills, as well as an established link to where and when in the game context they would be used. Dunn (2006) also proposed that the level of tactical knowledge in novice performers and their DM ability could exceed their technical ability to execute these skills in a performance context. This further reinforces the importance of technical skills, which are continually emphasized by elite coaches. Indeed, we would suggest that the limited ability to execute skills influences the tactical options taken within a playing context. For example in field hockey, a player without the technical ability to produce an aerial pass would 
remove that tactical option; in short, they would not look for it so hardly ever see it. Similarly, the limited technical ability to pass using either hand in netball/basketball determines the tactical decision that is made. The way in which this technical deficiency impacts on DM has yet to be discerned although, anecdotally at least, it seems that players without a particular skill fail even to consider, let alone see the options related to their weakness. Unsurprisingly therefore, French, Spurgeon and Nevett, (1995) concluded that technical skill was a distinguishing factor in determining a player's performance level.

It therefore appears that the layering approach outlined in this paper presents a possible rationale for building decision-making knowledge (technical, tactical and strategic) as a mechanism to develop DM capabilities. Furthermore, the layering of information must consider the technical component performed within the tactical situation, so that both are contextualised within the performance vision (objective). In light of this, we propose that different theories located within the NDM paradigm could collectively (rather than singly) provide the best possible framework for developing team DM.

Pertinent to such challenges, RPD (Klein, 1998) proposes a dual system, which integrates intuition and subjective analysis of the situation. The application of RPD (see also Klein, 1993) to team sports enables us to gain an understanding of what cues are attended to, together with how they are prioritised and used to inform and influence decisions. In field hockey for example, at a basic level the cues from where an attacker is carrying the ball and the angle of the stick will inform the defender of potential moves that the attacking player might take. Hence, these cues facilitate decisions as to what actions the defender might initiate, such as which technique for tackling will be implemented. At a more complex level these technical cues, combined with identified elements from the tactical environment (thus combining layers 2 and 3 of the DM process), would inform what decisions might be taken at an individual level and collectively at a defensive team level (action involving multiple players). By contrast, a reliance on a cue-only driven approach (bottom-up) would often result in DM errors such as defending the option being 
'shown' by the attacker but permitting a pass which carries greater threat to the team. Clearly, reliance on intuition alone is too risky as pattern matching can generate flaws in perception (Klein, 2008).

Klein's (1993) cue based RPD model incorporates both intuition and analysis of situations (Klein, 2008) and can be used to some extent but not solely, to explain basic DM in sport. However, as proposed in the first model (Figure 1) there also needs to be an established link of technical skill proficiency to the competitive context (Bock-Jonathon, Venter, \& Bressen, 2007). This lends additional support to the layering of technical, tactical and strategic approaches as proposed in this paper.

\section{Tactical and situational awareness development: Layer 3.}

This third layer of the model indicates the development of cognitive knowledge structures (MMs and eventually SMMs) relating to tactical play, which incorporate situational factors and visual cues. DM at the expert level requires both individuals and teams to adapt their knowledge to the complexity of the situation in which they are playing (termed macro-cognition; see Klein 2008). Making effective decisions requires MMs to be constructed that are unique to specific situations (e.g., attacking play from the left defense). The nature of the situation (for example attacking verses defensive situations) will require a different DM engagement. Such cognitive representations are driven by the experience of the player and the playing philosophy established by the team (utilising top-down knowledge processing). This process occurs simultaneously, as the player is actively perceiving their current performance context (location on field, position of teammate and opposition, plus other contextual factors); making sense of the current performance situation they are participating in (bottom-up knowledge processing) within the context of the team playing philosophy (top-down knowledge processing). To gain an understanding of these concepts within the performance context warrants the additional inclusion of a situational 
awareness framework as a theoretical approach to justify the importance of the environment in which decisions are made.

Situational Awareness (SA) proposes a hierarchical model (Endsley \& Garland, 2008) consisting of three levels. Level 1 involves the perception of important cues. Level 2 is concerned with the comprehension of these cues and level 3 allow individuals to predict future situations by integrating past experience to the situation. Caserta and Singer (2007) proposed that level 3 Situational awareness distinguishes elite from non-elite performers in any domain.

Pertinently for applied practice, this paper proposes that such situational specific knowledge structures (MMs/SMMs) are developed through the combined interaction of off-field, slow reflective deliberate environments (team meetings etc.) and on-field dynamic training/matches environment (see Figure 2). The development of MMs for specific situations enables players and teams to attend to information that is agreed as being significant. Players then prioritise and order this information so that the correct course of action can be followed (Bate \& Richards, 2011; Thevenot, 2009).

As a further element, combining the contributions of RPD and SA, Klein et al's (2007) concept of sensemaking makes a valuable contribution to enhancing our understanding of how MMs are developed. Sensemaking goes beyond the comprehension of environmental cues and the reader is encouraged to read Klein et al, (2007) for a comprehensive account of The Data-Frame Sensemaking Theory. Sensemaking proposes an approach in which the experience of the individual (we propose also the experience of the team) can be used to 'frame' (comprehend) a playing situation. As the players and team collectively 'frame' the situation (place it in context of previous experiences), data points (performance cues) in competition can be interpreted and collectively responded to. Sensemaking facilitates the performer (we argue also the team) establishing connections and relationships between environmental cues. Such visual perceptions are contextualised within previous playing experience (individual/team). The construction of slow, deliberate, learning situations, 
whereby individuals are empowered to reflect and contribute to providing a solution (Figure 2), results in the content of these situations being internalised and stored by players/teams (Richards et al., 2009, 2012). This produces an increasingly robust mental model where, in future situations, information perceived in the environment is matched, enabling rapid execution of technical and tactical skills (Bate \& Richards, 2011).

While we await investigation of sensemaking in sport, the implication for coaches is that the information necessary to formulate MMs and SMMs requires development in a progressive and logical manner. This will incorporate the perception of the situation together with cue recognition. As generations of the playing concepts develop, both in variation (specific to situations) and detail, the playing philosophy of the team moves from providing the simplest answer to a more complex, strategy-based approach as multi-play patterns are developed. In this regard, the update of information we gain relating to MMs enables the models to be modified and refined in light of new experiences (Kessler, Duwe, \& Strohmer, 1999). The engagement in slow deliberate reflective processes during off-field team meetings not only updates team MM/SMMs but creates a simulation environment where solutions are discussed which relate to future playing situations. This team simulation process (Klein, 2008) contributes to an improvement in the consistency of team decision in a competitive context. It is a crucial facet through which SMMs are developed as genuinely shared, rather than just a conglomeration or averaging of different individuals viewpoints. In short, what is developed is a real shared vision - a team vision rather than some political compromise. The team parameters which impact on shaping the development of a team's vision are multifactorial (see Richards et al 2009) and include player/team maturity, empowerment process and experience to mention only a few.

\section{Strategic development: Layer 4.}

In the fourth stage of the model, team members are perceiving the situation (perceptual cues, knowledge structures) in the same way by placing the same value on key markers (cues) in the environment. Through having a common perception, the team can now generate a reduced 
number of plausible options (playing actions) by having a commonly agreed assessment of the situation. Where an obvious playing option has been perceived this will be taken. Difficulty arises when several options present themselves which all relate to achieving the same team outcome. In such situations we would argue that the agreed team SMM (players perceiving the situations the same) results in the team intuitively considering perhaps only one or two possible options. Notably, however, this intuitive understanding has arisen from the slow deliberate reflective team meetings and associated discussion. Thus, through 'satisficing' (see Simons, 1957), the team collectively takes an option, which will produce a successful outcome.

The final challenge for sports coaches developing DM skills in a team context is the construction of communication and coordination processes. The success of team performance is reliant on tasks being simultaneously performed by multiple or cooperating individuals. Therefore, individual's tasks, language, thinking and schema need to be coordinated and integrated (Eccles \& Tenenbaum, 2004). In team sport, success will frequently be determined, not only by the contribution of the team member's skill-sets (layer 2) but also through the coordinated and integrated manner in which the perception, decisions and actions of the team are executed. In order for the team to perform in this coordinated manner, members must share a common perception of the objectives of the task (game plan) and the approaches required to achieve success (team principles of play). This shared approach or common way of thinking is developed through SMMs and represents the highest strategic level of DM required at the elite levels in team sports.

Although SMMs have been recognised as a method for studying skilled performance in teams (Cannon-Bowers, Salas, \& Converse, 1990), the exploration of SMMs in a sporting context has been limited. Yet it seems logical that the integration of multiple tasks and roles of individuals need to be communicated and coordinated in environments where the dynamic nature and speed of task execution limits discussions. At the same time, situations must be similarly perceived if they are to be collectively responded to in order to achieve the team's objective. Bridging this 
apparent conundrum, SMMs provide a structure for teams to share a common perception of the expected outcome of a situation (winning a game) and the process required to achieve this (understanding of the coordination of individuals' roles). SMMs also act to speed-up and ease communication. For example, Heath (1991), working with a baseball team, highlighted how developing SMMs reduced the need for prolonged and explicated communication within the team.

\section{Beta vision of performance: Layer 5.}

When collectively performed, the development of the SMM at the strategic level results in delivery of the beta-version of performance in the competitive setting (Richards et al, 2009). This will, in theory, consistently produce the 'ideal' performance. Of course, this vision of performance is continually being reshaped and developed by coaches and players throughout the duration of the team's life cycle. The extent to which this final stage is successful is very much influenced by the quality of the previous layers of tactical recognition, technical execution of skills and the development of shared cognitive frameworks. In practical terms, some would propose that it is rarely, if ever, achieved. However, pursuit of this ideal through the methods described above is arguably the best way in which high levels of performance can be realised.

\section{Model 2 - Developing rapid high-pressure decision-making through slow deliberation}

Figure 2 presents a dual process model in which rapid, high-pressured, team DM can be developed in dynamic competitive situations, through slow deliberate conscious off-field learning combined with on field experience in games and training. The model has been empirically tested and proved successful in field-hockey (Richards et al., 2009); football (Bate \& Richards, 2011; Merola \& Richards, 2010) and netball (see Richards et al., 2012). The model proposes that, in order to develop team DM skills, practitioners need to develop dual learning systems which incorporate a slow deliberate reflective environment (off-field) and a dynamic performance environment (on-field) where applied experience and knowledge is ascertained and 'automated'. 
432

433

434

435

436

437

438

439

440

441

442

443

444

Both of these environments integrate the knowledge structures presented in the first section of this paper.

Such dual processes draw parallels and relevance to other DM research situated outside of sport in two respects. Firstly, there is considerable evidence that people make decisions by utilising their previous experiences (Klein, 2008; Lipshitz, 1993). The recognition that an individual uses previous experiences to make decisions in real-world situations is reliant on both their perception and recognition of the situation. Secondly, the proposal that DM requires dual systems, which are responsible for directing attentional focus has been substantially investigated (Epstein, 1994; Evans 2008). Examples include Kahneman (2003) proposing his System 1 and System 2; and perhaps more significantly in context of this paper, the work by Eysenck, Derkshan, Santos and Calvo (2007) on Attentional Control Theory. Both of these approaches propose that decisions cannot be made solely on the intuitive instincts of an individual. The process ideally involves both intuitions and analysis of the situation (a dual system).

Eysenck et al. (2007) also made reference to a dual system and proposed that individuals have a goal-directed attentional system and a stimulus driven system. In pressurised situations, according to Eysenck et al. (2007), there is less reliance on the goal-directed attention system (our equivalent of the top-down system) and more reliance on the cue driven system (cf. Bishop, Duncan, Brett, \& Lawrence, 2004) to shape decisions (our bottom-up approach). This would explain the decrease in quality of DM evident in highly pressurised matches, supporting the need for a dual system. In these circumstances, players often revert back to processing instant cues in their environment, which is often less helpful as it is not informed by the large context (game objectives) in which the decision is made. So there appears to be growing and independent support for the principle that the complexity of DM requires more than one system to process information. 
Model 2 illustrates the cyclic link where knowledge is developed simultaneously in both off-

457

field and on-field environments (dual process). The integration of the off-field environment (e.g., team discussion and reflection) into the coaching process, in which performers are empowered to discuss and explore aspects relating to play; combined with on-field experiences results in the development of enhanced team DM. The cycle relationship between on-field and off-field environments facilitates the development of a team direct stimulus system (off-field) and an intuitive experienced based system (on-field) team decision making framework. The off-field environment facilitates the development of robust SMM which players personalised to their playing position and in doing so construct internalised plans (understanding of their own role in that specific situation, Richards et al, 2009). The establishment of the SMM and internalised plans are utilised in future playing situations, allowing deployment of enhanced rapid DM skills (Richards et al., 2012).

Model 2 (see Figure 2) presents a three phase approach to developing team DM. The model incorporates both feedback and feed-forward mechanisms, creating a cyclic link between continual learning and the evolution of playing constructs. The three phases are distinctly different and are responsible for developing cognitive structures (MMs/SMMs outlined in model 1) in relation to the specific performance contexts. The creation of these knowledge structures facilitates the players (individually or collectively) attending to agreed information in the performance environment. Once information is attended to, it is prioritised and ordered in relation to agreed principles of play, enabling the correct action of play to be executed individually or collectively as a team.

\section{[INSERT FIGURE 2 HERE]}

\section{Model 2: Phase 1}

The first phase of the model highlights the establishment and generation of playing concepts. The complexity of these concepts (MMs) will very much depend on the performance level parameters of the individuals and the team. Constructing a shared understanding of the team's 
482

483

484

485

486

487

488

489

490

491

492

493

494

495

496

497

498

499

500

501

502

503

504

505

506

507

objectives allows the development of team principles. These principles also allow the concepts to be recognised in play and are essential in establishing subsequent SMMs. Establishing a shared player perception of playing concepts (playing philosophy) and the generation of playing principles that underpin these playing concepts, results in the development of a shared language that can be used to further develop teamwork (Mascarenhas \& Smith, 2011).

\section{Model 2: Phase 2}

The second phase of the model relates to the development and enrichment of knowledge structures. A combination of empirical and experiential evidence suggests that, in order to develop rapid DM skills in individuals and teams in competitive situations, the process of slow, deliberate learning is required. Without this, developing a team's understanding of concepts and transferring such knowledge to competitive situations is limited, resulting in a 'recipe' or 'Standard Operational Procedure' style of DM, often choreographed by the coach.

Most playing concepts (phase 1) are developed through team meetings of various styles, where the use of video and performance analysis is increasingly common. In most situations, clips are presented to the players where aspects relating to the video are reviewed. Through slow deliberate and conscious team discussions, the video is explored and a group decision is made with regard to the best option to take. Reflecting on these situations and identifying the important aspects of each clip enables SMMs to be constructed. This employs a slow deliberate approach to DM to develop a subsequent NDM application in the performance setting. Engaging in such slow deliberative processes enables similar situations in future games to be responded to at a quicker rate and more successfully (cf. Mascarenhas, Collins, Mortimer, \& Morris, 2005).

The creation of this slow, deliberate environment must also address the development of knowledge structures that are specific to the situation. Reviewing the situation at a descriptive level is not sufficient to improve DM. The development of more elaborate, often multiple 
option SMMs for specific situations can more effectively guide the attention of the performer to the relevant aspects of the display. This shared perception also facilitates a collective approach to the situation where individuals have a clear understanding of their own role in the situation, as well as the action required by others within the context, in addition to understanding the actions being performed by the opposition. A shared perception and common understanding of situations produces a higher level of connectivity between players as well as an agreed team approach to addressing the situation. This also leads to the development of a common language, enabling players to have an agreed understanding of a situation where the execution of action by multiple players can be verbalised in just one or two words (Richards et al., 2012).

As the playing concepts develop both in variation (specific to situations) and detail, a parallel development occurs with established, team specific SMMs. Original, simple SMMs develop in complexity to match the increasing complexity of multiple patterns of tactical play. This deliberate knowledge environment then shapes both the development and interaction of offfield and on-field practices.

\section{Model 2: Phase 3}

In the third phase of the model, the cognitive structures are applied to the competitive situation. The application of these structures to the performance setting enables individuals to execute technical and tactical skills successfully. Additionally, the team specific SMM facilitates greater and more effective connective play. This is evident in multiple play patterns being created by teams. The shared perception of the preferred option is recognised and communicated by teammates, resulting in coordinated actions of multiple players.

The link between the slow deliberate environment (where constructs are created) and the applied environment (where the constructs are executed) is facilitated by an interacting pair of feed-forward and feedback mechanisms. These mechanisms enable the complexity of SMM to be developed as the team progresses to higher playing standards. The mechanisms also 
provide a process where the models are continually evaluated for their effectiveness, as both

535 the individual and the team reflect both on and in action.

\section{Conclusion and moving to the next step}

537 In this paper we presented an empirically tested framework for developing team decision making. The model has been developed over 15 years of elite coaching practice and is informed and influenced by both academic research and practical understanding. We apologise if the level of referencing is a distraction to readers but, we suggest, the complexity

541 of team decision making requires the integration and understanding of several key concepts, each originating from a specific and different discipline. Each of these disciplines (motor control, social psychology, cognitive psychology, NDM and pedagogical preprocess) have influenced our understanding of how team decision making is developed. Unfortunately, 545 however, many authors (both applied and academic) have to date explored decision making 546 through use of these various disciplines in isolation. We have focused not just on the messages from several disciplines but, more particularly, on their integration; considering the interactive and complimentary influences as well. We commend this approach to practitioners and researchers alike.

In summary this paper presented a five stage framework which incorporates a holistic approach to developing team decision making. The paper addresses the development of rapid high speed DM processes (in competition) whilst integrating the development of SMMs and cohesive structures within the team's social milieu. Reflecting this essential complementarity, we suggest that the development of individual and team DM skills in sport cannot be developed effectively without the use of a slow, deliberate, off field reflective environment 556 and the application of this slow deliberate thinking into the applied tactical knowledge 557 environment (performance context). The decision making process is complex and multifactorial as it involves classical and naturalistic approaches; on-field and off-field 
560

561

562

563

564

565

566

567

568

569

570

571

572

573

574

575

576

577

578

579

580

581

that much of what is proposed here has been tested empirically in high level sport. This is a genuinely workable solution to a real life issue. By providing coaches with the step-by-step logic through which the model has been derived, we hope that the understanding of these parallel processes has been developed to the degree necessary to enable them to take and apply the model in their own team settings.

\section{References}

Bar-Eli, M., Plessner, H., \& Raab, M. (2011). Judgment decision making and success in sport. West Sussex, UK: John Wiley \& Sons, Ltd.

Bate, B., \& Richards, P. (2011, April). Developing team decision making capabilities in a professional football team. Paper presented at British Association of Sport and Exercise Sciences National Student Conference, Chester.

Beilock, S. L. (2009). Grounding Cognition in action: Expertise, comprehension and judgment. In M. Raab, J.G. Johnson, \& H.R. Heekeren (Eds.), Progress in brain research (Vol 174, pp. 3-11). New York, NY: Elsevier.

Bishop, S., Duncan, J., Brett, M., \& Lawrence, A.D. (2004). Prefrontal cortical function and anxiety: Controlling attention to threat-related stimuli. Nature Neuroscience, 7, 184-188.

Bock-Jonathon, B. B., Venter R. E., \& Bressen, E. S. (2007). A comparison between skill and decision making ability of netball players at club level: Pilot work. South African Journal for Research in Sport, Physical Education and Recreation, 29 (1), 29-38.

Caserta, R. J., Young, J., \& Janelle, C. M. (2005). Old dogs, new tricks: Training the perceptual skills in senior tennis players. Journal of Sport and Exercise Psychology, 29 (4), 479-497. 
582

583

584

585

586

587

588

589

590

591

592

593

594

595

596

597

598

599

600

601

602

603

604

Caserta, R. J., \& Singer, R. N. (2007). The effectiveness of situational awareness learning in response to video tennis match situations. Journal of Applied Sport Psychology, 19 (2), 125-141.

Cannon-Bowers, J. A., Salas, E., \& Converse, S. A. (1990). Cognitive psychology and team training: Training shared mental models of complex systems. Human Factors Society Bulletin, 33(12), 1-4.

Dunn, J. (2006). Coaching decision making in rugby. Retrieved 10 May 2006, from http://www.rugby.com.aucomminity_rugbycoachinglevel_iii_papers_decision_making,30435.html .

Eccles, D. W., \& Tenenbaum, G. (2004). Why an expert team is more than a team of experts: A cognitive conceptualization of team coordination and communication in sport. Journal of Sport and Exercise Psychology, 26, 542-560.

Endsley, M.R., \& D.J. Garland. (2008). Situational awareness analysis and measurement. New York, NY: CRC Press.

Epstein, S. (1994). "Integration of the Cognitive and Psychodynamic Unconsciousness". American Psychologist, 49, 709-724.

Evans, J. (2008). “Dual Processing Accounts of Reasoning, Judgments and Social Cognitions”. Annual Review of Psychology, 59, 255 - 278.

Eysenck, M. W., Derakshan, N., Santos, R., \& Calvo, M. G. (2007). Anxiety and cognitive performance: Attentional control theory. Emotion, 7, 336-353.

French, K., Spurgeon, H., \& Nevett, M. (1995). Expert-novice difference in cognitive and skill execution components of youth baseball performance. Research Quarterly for Exercise and Sport, 3, 194-201. 
605

606

607

608

609

610

611

612

613

614

615

616

617

618

619

620

621

622

623

624

625

626

627

628

Heath, S.B. (1991). “It's about winning!” The language of knowledge in baseball. In L. Resneck, J. M. Levine, \& S. Behrend (Eds.), Shared cognition: Thinking as social practice (pp. 101-124). Washington, DC: American Psychological Association.

Kahneman, D. (2003). Maps if bounded reality: Psychology for behavioural economics. American Economic Review, 93(5), 1449-1475.

Klein, G. A. (1993). Recognition Primed Decision (RPD) model of rapid decision making. In G. Klein, J. Orasanu, R. Calderwood, \& C. E. Zsambok (Eds.), Decision making in action: Models and methods (pp.138-147). Norward, NJ: Ablex.

Klein, G. (2008). Naturalistic decision making. Human Factors, 50(3), 456-460.

Klein, G. (1998). Source of power: How people make decisions. Cambridge, MA: MIT Press.

Klein,G., J.K Phillips, E.L Rall, \& D.A. Peluso. (2007). “A data-frame theory of sensemaking". In R. Hoffman (Ed.), Expertise out of context. Proceedings of the sixth international conference on naturalistic decision making (pp. 113 - 155). New York, NY: Taylor and Francis.

Kessler, K., Duwe, I., \& Strohner, H. (1999). Grounding mental models - Sub-conceptual dynamics in the resolution of linguistic reference in discourse. In R. Hoffman (Ed.), Expertise out of context. Proceedings of the sixth international conference on naturalistic decision making (pp. 219 - 262). New York, NY: Taylor and Francis.

Lipshitz, R. (1993). Converging themes in the study of decision making in naturalistic settings. In G. Klein, J. Orasanu, R. Calderwood, \& C. E. Zsambok (Eds.), Decision making in action: Models and methods (pp. 103-137). Norward, NJ: Ablex.

Mascarenhas, D. R. D., Collins, D., Mortimer, P., \& Morris, R. L. (2005). Training accurate and coherent decision-making in rugby union referees. The Sport Psychologist, 19, 131147. 
629

630

631

632

633

634

635

636

637

638

639

640

641

642

643

644

645

646

647

648

649

650

Mascarenhas, D. R. D., \& Smith, N. C. (2011). Developing the performance brain: Decision making under pressure. In D. Collins, A. Button, \& H. Richards (Eds.), Performance Psychology (pp. 245-268). London, UK: Elsevier.

Merola, T., \& Richards, P. (2010, April). Developing Decision Making Skill in youth footballers. British Association of Sport and Exercise Sciences National Student Conference, Aberystwyth.

North, J. S., \& Williams, A. M. (2008). Identifying the critical time period for information extraction when recognising sequences of play. Research Quarterly for Exercise and Sport, 79, 268-273.

Oranasanu, J. \& Salas, E.(1993). Team decision making in complex environments. In G.A. Action: Models and methods, (pp.327 - 345). Norwood, NJ: Ablex.

Richards, P., Mascarenhas, D. R. D., \& Collins, D. (2009). Implementing reflective practice approaches with elite team athletes: parameters of success. International Journal of Reflective Practice, 10(3), 353-363.

Richards, P., Collins, D. \& Mascarenhas, D. R.D. (2012). Developing rapid high-pressure team decision-making skills. The integration of slow deliberate reflective learning within the competitive performance environment: A case study of elite netball. International Journal of Reflective Practice, 1-18. DOI: 10.1080/14623943.2012.670111

Ross, K. G., Battaglia, D. A., Phillips, J. K., Domeshek, E. A., \& Lussier, J. W. (2003). Mental models underlying tackling thinking skills. . Retrieved November 18, 2012, from http://www.peterhancock.ucf.edu/Downloads/humanfactors_2/Expertise\%20and\%20Deci sion-Making/Expertise\%20Lecture\%20KRoss.doc. 
651

652

653

654

655

656

657

658

659

660

661

662

663

664

665

666

667

668

Rouse, W. B., Cannon- Bowers, J. A., \& Salas, E. (1992). The role of mental models in team performance in complex systems. IEEE Transactions on Systems, Man and Cybernetics, 22, 1296-1308.

Simons, H.A. (1957). Models of Man: Social and Rational. New York: Wiley.

Serfaty, D., Macmilan, J., Entin, E. E, \& Entin, E. B. (1997). The decision making expertise of battle commanders. In C. E. Zsambok, \& G. A. Klein, (Eds). Naturalistic decision making (pp. 233-246). Mahwah, NJ: Lawrence Erlbaum.

Starkes, J. L. \& Ericson, K. A. (2003). Expert performance in sports: Advances in research on sport expertise. Champaign, IL: Human Kinetics.

Thevenot, C. (2009). Arithmetic word problem solving: evidence for the construction of mental models, Acta Psychological, 133, 90-95.

Weick, K.E \& Roberts, K.H. (1993). Collective mind in organizations: Heedful interrelating on flight decks. Administrative Science Quarterly, 38, 357 - 381.

Westbrook, L. (2006). Mental models: A theoretical overview and preliminary study. Journal of Information Services, 32, 563-579.

Williams, A. M. (2009). Perceiving the intentions of others: how do skilled performers make anticipation judgments? In M. Raab, J. G. Johnson, \& H. R. Heekeren (Eds.), Progress in brain research (pp. 73-83). New York: Elsevier. 